\title{
Effects on magnetic reconnection of a density asymmetry across the current sheet
}

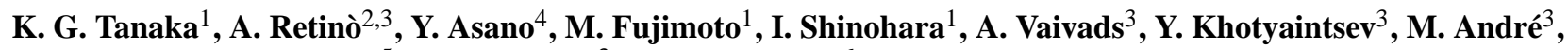 \\ M. B. Bavassano-Cattaneo ${ }^{5}$, S. C. Buchert ${ }^{3}$, and C. J. Owen ${ }^{6}$ \\ ${ }^{1}$ Japan Aeronautical Exploration Agency, Kanagawa, Japan \\ ${ }^{2}$ Space Research Institute, Austrian Academy of Sciences, Graz, Austria \\ ${ }^{3}$ IRFU, Uppsala, Sweden \\ ${ }^{4}$ Tokyo Institute of Technology, Japan \\ ${ }^{5}$ IFSI-INAF, Roma, Italy \\ ${ }^{6}$ MSSL, London, UK
}

Received: 27 December 2007 - Revised: 9 June 2008 - Accepted: 17 July 2008 - Published: 15 August 2008

\begin{abstract}
The magnetopause (MP) reconnection is characterized by a density asymmetry across the current sheet. The asymmetry is expected to produce characteristic features in the reconnection layer. Here we present a comparison between the Cluster MP crossing reported by Retinò et al. (2006) and virtual observations in two-dimensional particle-in-cell simulation results. The simulation, which includes the density asymmetry but has zero guide field in the initial condition, has reproduced well the observed features as follows: (1) The prominent density dip region is detected at the separatrix region (SR) on the magnetospheric (MSP) side of the MP. (2) The intense electric field normal to the MP is pointing to the center of the MP at the location where the density dip is detected. (3) The ion bulk outflow due to the magnetic reconnection is seen to be biased towards the MSP side. (4) The out-of-plane magnetic field (the Hall magnetic field) has bipolar rather than quadrupolar structure, the latter of which is seen for a density symmetric case. The simulation also showed rich electron dynamics (formation of fieldaligned beams) in the proximity of the separatrices, which was not fully resolved in the observations. Stepping beyond the simulation-observation comparison, we have also analyzed the electron acceleration and the field line structure in the simulation results. It is found that the bipolar Hall magnetic field structure is produced by the substantial drift of the reconnected field lines at the MSP SR due to the enhanced normal electric field. The field-aligned electrons at the same MSP SR are identified as the gun smokes of the electron acceleration in the close proximity of the X-line. We have also analyzed the $\mathrm{X}$-line structure obtained in the simulation to find that the density asymmetry leads to a steep density gradi-
\end{abstract}

Correspondence to: K. G. Tanaka

(tkentaro@stp.isas.jaxa.jp) ent in the in-flow region, which may lead to a non-stationary behavior of the X-line when three-dimensional freedom is taken into account.

Keywords. Magnetospheric physics (Magnetopause, cusp, and boundary layers) - Space plasma physics (Charged particle motion and acceleration; Magnetic reconnection)

\section{Introduction}

Magnetopause (MP) is known as the interface between the intrinsic terrestrial magnetic field and the interplanetary magnetic field (IMF) in the solar wind. Bounded by the magnetopause, the cold-dense solar wind and the hot-tenuous magnetospheric (MSP) plasmas come in contact with each other. It is well known that energy and momentum of solar wind plasmas are allowed to enter the MSP by way of magnetic reconnection at MP (e.g. Paschmann, 1979). This importance in the global scale context is one of the reasons that MP reconnection has been studied extensively (e.g. Phan et al., 2000).

The observations suggest that MP reconnection location varies according to the IMF condition (e.g. Pinnock et al., 2003; Fuselier et al., 2005; Trattner et al., 2007). The wellknown fact is that the reconnection site is shifted to poleward of the cusp under northward IMF. The aurora spot in the cusp is one of the clearest evidence for the direct injection of the solar wind ions along the reconnected field lines (Phan et al., 2003; Frey et al., 2003). Variation of the upstream condition provides various setting to study reconnection physics using data obtained at MP and this is another reason why MP reconnection has been studied extensively. Yet another reason for studying reconnection at MP is that the MP crossing

Published by Copernicus Publications on behalf of the European Geosciences Union. 
provides a simpler cut through a reconnection layer than a magnetotail case because the MP has a sharp density gradient and/or magnetic field structures. That feature enables us to embed with more ease the observed smaller-scale structures in a larger-scale context.

Indeed recent in-situ observations at MP have been showing hint of electron scale physics embedded in large-scale reconnection dynamics. The observation of the subsolar MP crossing by the Polar spacecraft under the southward IMF condition (Mozer et al., 2002) has shown a textbook-like example of the MP structure with (1) clear signatures of the Hall current system in the ion diffusion region, (2) non-zero normal component of the magnetic field, (3) possible signature of the electron diffusion region in the decoupling of the electron flow from the $\boldsymbol{E} \times \boldsymbol{B}$-drift, and (4) the separatrix on the MSP side showing the low frequency electric field turbulence, the parallel electric field, and the local minima in the plasma density. Cluster observations (André et al., 2004; Panov et al., 2005; Stenberg et al., 2005) have shown the electron scale current layers of a few electron inertial scale widths associated with currents and strong electric fields on the magnetospheric side of the MP.

The magnetotail is another important region in the context of magnetic reconnection. In this case, the reconnecting magnetic field consists of a pair of magnetospheric plasmas having symmetric density profile along the current sheet normal direction. Interests for most of simulationists tended to focus on magnetotail-like configurations (e.g. GEM challenge - Birn et al., 2005; Newton Challenge - Birn et al., 2006) probably because the settings are less complicated than the MP case. This, however, does not mean that MP-like cases are just complicated and does not contain any interesting plasma physics associated with it.

Because of the contact between the solar wind plasmas and the magnetospheric plasmas, a large density gradient is formed at the magnetopause. In addition to the various magnetic field settings, the density asymmetry should be regarded as one of the most characteristic features of MP current layer. In such a condition, the lower-hybrid drift instability (LHDI) may be driven unstable and has been the target of simulation studies. Gary and Sgro (1990) have firstly carried out simulation study on the LHDI with MP-like situation. After Gary and Sgro (1990), Scholer et al. (2003), Ricci et al. (2004), Daughton et al. (2004), Shinohara and Fujimoto (2005), Tanaka et al. (2004, 2005, 2006) and Silin and Büchner (2006) have proposed a new view at the possible role of LHDI at the edges.

Nakamura and Scholer (2000) performed hybrid simulations (mass-less electrons) and studied another aspect of the density asymmetry, that is, its impact on the two-dimensional reconnection dynamics at MP. They showed that the Hall magnetic field $\left(B_{M}\right)$ has the bipolar structure rather than the quadrupolar structure seen in a density symmetry case.

Swisdak et al. (2003) studied the effects of the plasma pressure gradient in the presence of non-zero guide field by two-dimensional full-particle simulations. In the case studied, the magnetic pressure had different values on different sides and the total pressure balance is maintained by different plasma pressures on different sides (As will be shown, the case studied in the present paper has zero-guide field and equal magnetic/plasma pressure across MP.). In this case the $\mathrm{X}$-line slides along the current sheet due to diamagnetic drift of the electrons and, when the $\mathrm{X}$-line sliding speed is larger the reconnection rate is found to be smaller.

Borovsky and Hesse (2007) have examined the MP-like reconnection between two plasmas with different mass densities using two-dimensional resistive magneto-hydro dynamics (MHD) simulations. Their systematic survey has shown that the reconnection rate is scaled by the hybrid Alfvén speed. They have found that the plasma outflow from the reconnection region becomes larger and more stretched on the low mass density side than on the high mass density side. Cassak and Shay (2007) have applied a Sweet-Parker scaling analysis to asymmetric anti-parallel reconnection in which both the plasma number densities and magnetic field strengths are different between the MSP and magnetosheath (MSH). They have found that the location of the X-line decouples from the stagnant point. Then, they have also carried out two-dimensional resistive MHD simulation in order to verify their theoretical prediction and have shown that the decoupling of the $\mathrm{X}$-line from the stagnant point indeed takes place.

Recently Retinò et al. (2006) have presented detailed Cluster-II observations of a magnetic reconnection separatrix region on the magnetospheric side of the magnetopause (During ongoing reconnection at that MP, see Retinò et al., 2005). The observations were obtained poleward of the cusp under northward IMF and the magnetic field showed an approximately anti-parallel geometry (zero guide-field) with strong density asymmetry. In Retinò et al. (2006) three sub regions within the separatrix region on the MSP side of the MP were identified. The first subregion is a density dip adjacent to the magnetic separatrix. Inside the dip, a normal electric field directed to the center of the MP is enhanced. Both parallel and anti-parallel electron beams were detected. In the second subregion, plasma density increases gradually recovers to the MSP level while the electric field decreases. The electric field fluctuations at/below the electron plasma frequency were smaller in amplitude compared with the density dip region while sporadic emissions with narrow peaks around the electron plasma frequency appeared (suggesting ongoing electron-scale physics). In the third subregion, a sharp density gradient brought the density up to the MSH value and the electric field fluctuations show strong emissions around the lower-hybrid frequency range.

The results of Retinò et al. (2006) have motivated us to perform the simulation-observations synergy study described in the present paper. The characteristics of the event that caught our attention are: (1) The estimated distance from the X-line is rather small so that the reproduction of the observations by 


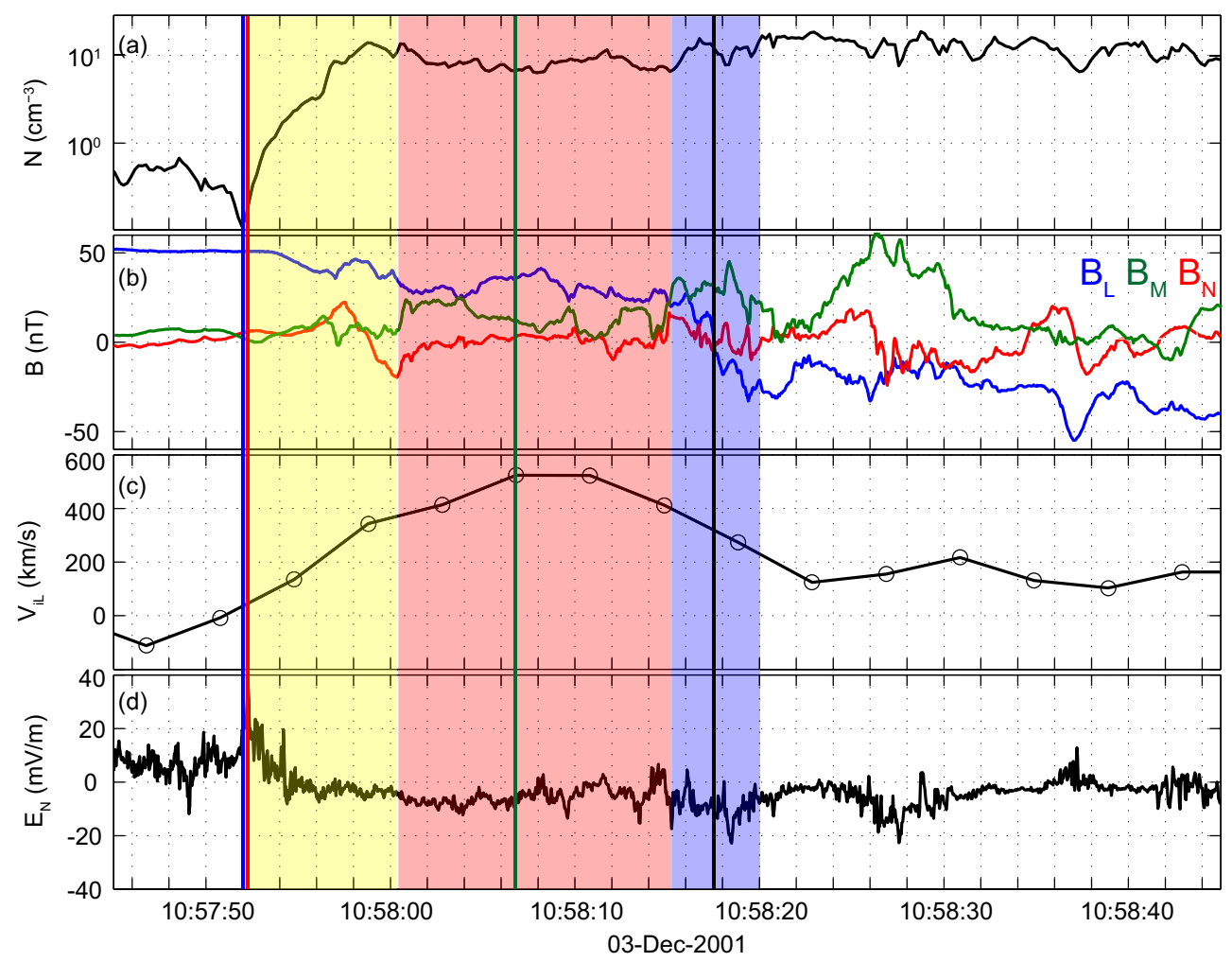

Fig. 1. Magnetopause crossing event observed by CLUSTER spacecraft (Retinò et al., 2006). (a) The electron number density $N_{e}$ obtained from the spacecraft potential, (b) three components of the magnetic fields, (c) the ion outflow bulk velocity $V_{i L}$, and (d) the normal component of the electric field $E_{N}$. The blue, red, green, and black lines show the locations of the electron density dip, $E_{N}$ peak, $V_{i L}$ peak, and the $B_{L}$ reversal, respectively. A deep electron density dip is seen at the MSP side SR, with a strongly enhanced positive $E_{N}$ peak. Ion outflow jet in the current sheet is biased toward the MSP side.

a full-particle simulation, whose box size is limited to less than $\sim 100$ ion-inertial length, is possible, (2) It is a largescale reconnection event in that ions are fully accelerated, (3) At the same time clear electron-scale signatures are noted if not fully resolved. Previous simulation-observation synergy studies seem to lack comparative investigation on the electron scale dynamics. On the contrary, the present paper will discuss the dynamics of the electron distribution functions, as well as the global structure. In this paper we will show that the reproduction of the key features obtained by Retinò et al. (2006) is fairly successful.

The structure of this paper is as follows: We first review Retinò et al. (2006) and point out the distinct features of the observed MP reconnection layer. Then the simulation model is described and the results are shown. In addition to the observation-simulation comparison, we will make detailed analyses of the two-dimensional MP structure in order to predict (1) how and where the electron acceleration takes place, and (2) the magnetic field structure, which enables us to understand the data along the spacecraft path (one-dimensional sampling) from a two-dimensional reconnection dynamics point of view. We also inspect (3) the density structure at the $\mathrm{X}$-line, and suggest possible effects in three-dimensional reconnection. Then, we will briefly discuss the effect of density profiles (symmetric or asymmetric) on the reconnection rate and saturation level.

\section{Key features in the observations}

\subsection{Overview of key features}

Figure 1 shows the MP crossing event around 10:58:00 UT on 3 December 2001 reported in Retinò et al. (2006). Shown in this figure are: (a) the electron number density $n_{e}$ obtained from the spacecraft potential, (b) three components of the magnetic fields, (c) the ion outflow bulk velocity $V_{i L}$, and (d) the normal component of the electric field $E_{N}$. Here the vector quantities are in the $\mathrm{LMN}$ coordinates, with $\mathrm{L}$ pointing to the magnetospheric field direction, $N$ to the MP normal direction (from MSP to MSH) and $M$ completing the righthanded coordinates.

The key features in the observations that are used to set the initial conditions of the simulation are (1) the reconnecting magnetic field is approximately anti-parallel and the magnitudes on both sides are almost the same. (2) The profile of plasma density is asymmetric, that is, the plasma density on 


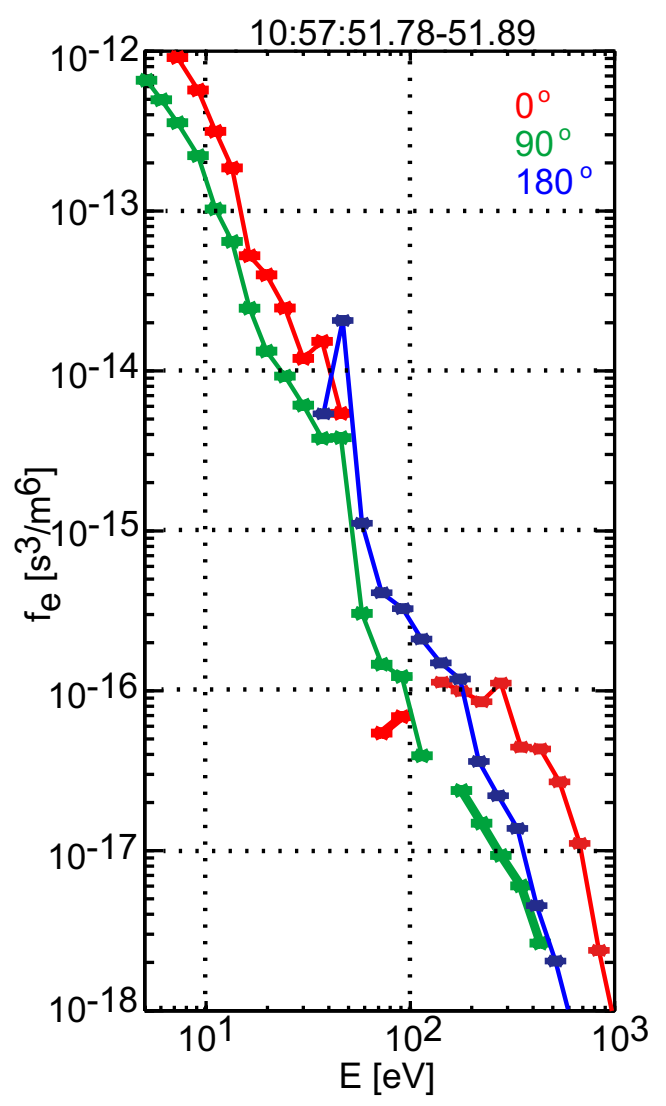

Fig. 2. Electron energy distribution function obtained at the density dip on the magnetospheric side of the magnetopause from 10:57:51.78 to 51.89 UT by the PEACE instrument. Two components of accelerated electrons are detected. One is the hot beam (red) in the parallel direction (away from the X-line) of a few hundreds $\mathrm{eV}$. The electron beam energy corresponds roughly to the local electron Alfvén velocity. The other is the colder population (blue) in the anti-parallel (into the X-line) direction.

the MSP side is $\sim 0.1$ of that on the MSH side. (3) The ion outflow in the magnetopause results from the reconnection jet. This information led us to design the initial setting to be described in detail later.

The features in the observations that are used to qualify the comparison with the simulation are as follows: (1) The plasma density dip is located at the separatrix on the MSP side. (2) The normal component of the electric field $E_{N}$ shows a localized positive peak at the density dip. (3) The reconnected ion flow is biased towards the MSP side. (4) The out-of-plane magnetic field $B_{M}$ (the Hall magnetic field) is positive, which is positive on the MSH side and negative on the MSP side for a symmetric reconnection.
2.2 The structure of the density dip on the magnetospheric side

At 10:57:52 UT, the plasma density depletes down to $\sim 0.02 \mathrm{~cm}^{-3}$, which is 0.2 times of the MSP value and 0.01 times of the MSH value. The location is adjacent to the separatrix of the magnetic field. In association with the dip, $E_{N}$ increases up to $\sim 40 \mathrm{mV} / \mathrm{m}$ in the positive direction. From 10:57:51.78 to 51.89 UT, that is, just around the density dip, two components of the accelerated electrons are detected by the Plasma Electron And Current Experiment (PEACE) instrument, which is shown in Fig. 2. One is a hot beam (red) in the parallel direction (away from the X-line) of a few hundreds $\mathrm{eV}$. The electron beam corresponds roughly to the local electron Alfvén velocity. The other is a cold population (blue) in the anti-parallel (into the $\mathrm{X}$-line) direction.

\subsection{The structure of the ion jet}

At 10:58:18 UT, the reconnecting magnetic field $B_{L}$ is reversed, meanwhile the peak of the ion jet is observed at 10:58:06 UT with $V_{i L} \sim 500 \mathrm{~km} / \mathrm{s}$. That is, the ion jet is biased toward the MSP side.

\subsection{The out-of-plane magnetic field component}

At 10:58:26 UT, when the spacecraft is on the MSH side, the magnetic field $B_{M}$ is enhanced up to $50 \mathrm{nT}$. Meanwhile $B_{M}$ at 10:58:04 UT in the MP is slightly increases up to $20 \mathrm{nT}$. That is, the out-of-plane field $B_{M}$ is only positive and is stronger on the MSH side.

\section{Simulation setup}

We use 2 1/2-dimensional (two spatial dimensions $\left(x_{N}, x_{L}\right)$ and three velocity components $\left.\left(v_{L}, v_{M}, v_{N}\right)\right)$ electromagnetic full particle simulation (Hoshino, 1987; Tanaka et al., 2004). In our simulations, plasmas consist of two populations. The Harris-type current sheet is used to ensure that the initial condition is not far from a Vlasov equilibrium. Full details are given below.

Two plasma populations are set in the initial condition. One population constructs the Harris current sheet (Harris, 1962). The Harris current sheet is expressed as $n_{C S}=n_{0} / \cosh ^{2}\left(x_{N} / D\right)$ for the plasma, and as $B_{L}=-B_{0} \tan h\left(x_{N} / D\right)$ for the magnetic field, where $D$ is the half-thickness of the current sheet. The plasma inside the current sheet has the ion-to-electron temperature ratio $T_{i, c s}=8 T_{e, c s}$. The other plasma population is the background plasmas having the number density of $n_{B G 1}=n_{1}\left\{1-1 / \cosh ^{2}\left(x_{N} / D\right)\right\}$ at $x_{N}>0$ (MSH side) and $n_{B G 2}=n_{2}\left\{1-1 / \cosh h^{2}\left(x_{N} / D\right)\right\}$ at $x_{N}<0$ (MSP side). It is distributed outside the current sheet. In the present paper, the background plasma density is set to $n_{1}=n_{0}$, and $n_{2}=0.1 n_{0}$, respectively. The background temperatures are 
Table 1. Comparison of ion and electron temperatures, the observed case versus the simulation.

\begin{tabular}{cccc}
\hline & & MSP & MSH \\
\hline \multirow{2}{*}{ Observation } & $T_{e}$ & $20-30 \mathrm{eV}$ & $50 \mathrm{eV}$ \\
& $T_{i}$ & $200 \mathrm{eV}$ & $50-200 \mathrm{eV}$ \\
\multirow{3}{*}{ Simulation } & $T_{e}$ & 1 & 1 \\
& $T_{i}$ & 19 & 1 \\
\hline
\end{tabular}

set to be $T_{i, B G 1}=T_{e, B G 1}=T_{e, B G 2}=T_{e, C S}$. Then, $T_{i, B G 2}$ is determined to conserve the pressure balance. After some easy calculation, the MSP side ion temperature is found to be $T_{i, B G 2}=19_{T e, C S}$. Using the MSH values, the initial plasma beta is found to be 0.29 .

Note that as was stated in Introduction, the initial pressure balance configuration is different from those of Swisdak et al. (2003). While the initial pressure balance of Swisdak et al. (2003) was done by varying magnetic field strength, that of the present case is done by varying the ion background temperature.

In Table 1, comparison of the ion and electron temperature between the observation and simulation is made. In this table, the MSP and MSH values are listed. the temperature units are electron volt $(\mathrm{eV})$ in the observation and $\left(T_{e, C S}\right)$ in the simulation, respectively. In the observation, a reference interval for the MSP domain is 10:55-10:56 UT, and for the MSH domain is 10:59-11:00 UT (not shown in Fig. 1). One should note that $T_{i, B G 2}=19$ in the simulation seems to be unrealistic. A brief discussion of the temperature difference between the observation and simulation will be made in the Discussion section.

In addition to the Harris magnetic field, a Gaussian-type perturbation vector potential $A_{M 0}=\varphi_{0} \exp \left[-\left(x_{N}^{2}+x_{L}^{2}\right) / 2 w^{2}\right]$ is introduced to set an $\mathrm{X}$-line at the center of the box. By setting $\varphi_{0}=e^{1 / 2} \delta B_{N} w, \delta B_{N}$ is the amplitude of the initial perturbation of the reconnected magnetic field. Here $\delta B_{N}=0.05 B_{0}$. Note that the initial guide field component is set $B_{M}=0$ in this study.

Hereinafter, magnetic field and density will be normalized by $B_{0}$ and $n_{0}$, respectively. The ion inertial length $\lambda_{i}=c / \omega_{p i}$ based on $n_{0}$ and the inverse ion gyro frequency $\Omega_{i}^{-1}$ based on $B_{0}$ are the units for spatial and time scales, respectively, and velocities will be measured in the MSH ion Alfvén speed unit $V_{A i}=B_{0} /\left(4 \pi m_{i} n_{0}\right)^{1 / 2}\left(m_{i}\right.$ : the ion mass). The electric field is normalized by $B_{0} \times\left(V_{A i} / c\right)$, where $c$ is the light speed. In this normalization, the MSH electron inertial length is $\lambda_{e}=M^{-1 / 2}\left(M\right.$ : ion-to-electron mass ratio $\left.m_{i} / m_{e}\right) . \quad M=100$ and thus $c / V_{A I}=10$ in our study.

We set the electron plasma frequency to electron gyro frequency ratio to be $\tau=\omega_{p e}=/ \Omega_{e}=1$ to reduce the computational cost, whereas the realistic value is $\sim 40$ in the magnetopause, and $\sim 5$ in the magnetotail. In the present study, we

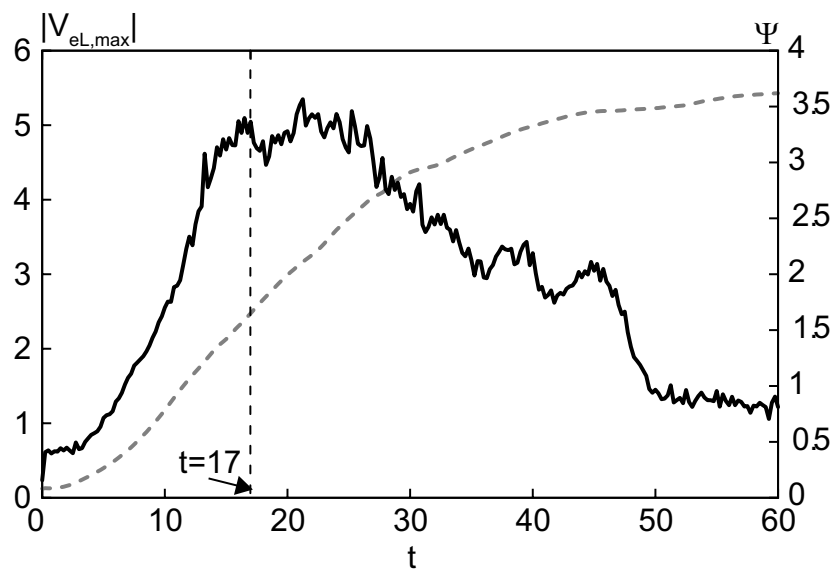

Fig. 3. Temporal developments of the reconnected magnetic flux $\Psi$, (dashed gray line with right vertical axis), and the maximum $\left|V_{e L}\right|$ (solid black line with left vertical axis) of the present simulation. At $t=17$, the reconnected is still growing and the electron jet has already reached maximum level.

do not expect $\tau$ to play a major role in our two-dimensional reconnection studies, as recently suggested by Haijima et al. (2008). In this paper, the dependence of reconnection rate and saturation level on $\tau$ has been surveyed using twodimensional full-particle simulation of density symmetric current sheet. Varying $\tau=1,2$, and 4 , they have shown that the saturation phase of the tearing mode is independent from $\tau$.

Periodic boundary conditions are imposed in the $x_{L}$ direction while conducting walls are set at the $x_{N}$ boundaries. The simulation box is $\left[0, L_{L}\right] \times\left[-L_{N} / 2, L_{N} / 2\right]$ in the normalized unit, with $L_{L}=24$ and $L_{N}=16$. The boundary conditions do not have any effects on the results shown in this paper. The half-thickness of the current layer $D$ is set 0.5 and the half width of the initial magnetic field perturbation $w$ is set 1 , respectively. The spatial grid of $\Delta x=0.9 \lambda_{D e}\left(\lambda_{D e}\right.$ : Debye length) and the time step of $\Delta t=0.15625 \Omega_{e}^{-1}\left(\Omega_{e}\right.$ : the electron gyro frequency) are adopted, respectively. 1024 particles per grid represent the unit density.

\section{Comparison between simulation and observation}

\subsection{Overview of simulational results}

Based on the key features stated in the previous section, we search for the time and location that are best compared with the observations. Figure 3 shows the temporal developments of the reconnected flux (solid black) and the maximum $\left|V_{e L}\right|$ (dashed black). At $t=17$ we found a situation which shows a fairly good agreement with the observation. It is found from Fig. 3 that at $t=17,(1)$ reconnection is still going on, and (2) a largely enhanced electron outflow jet is stably maintained. 


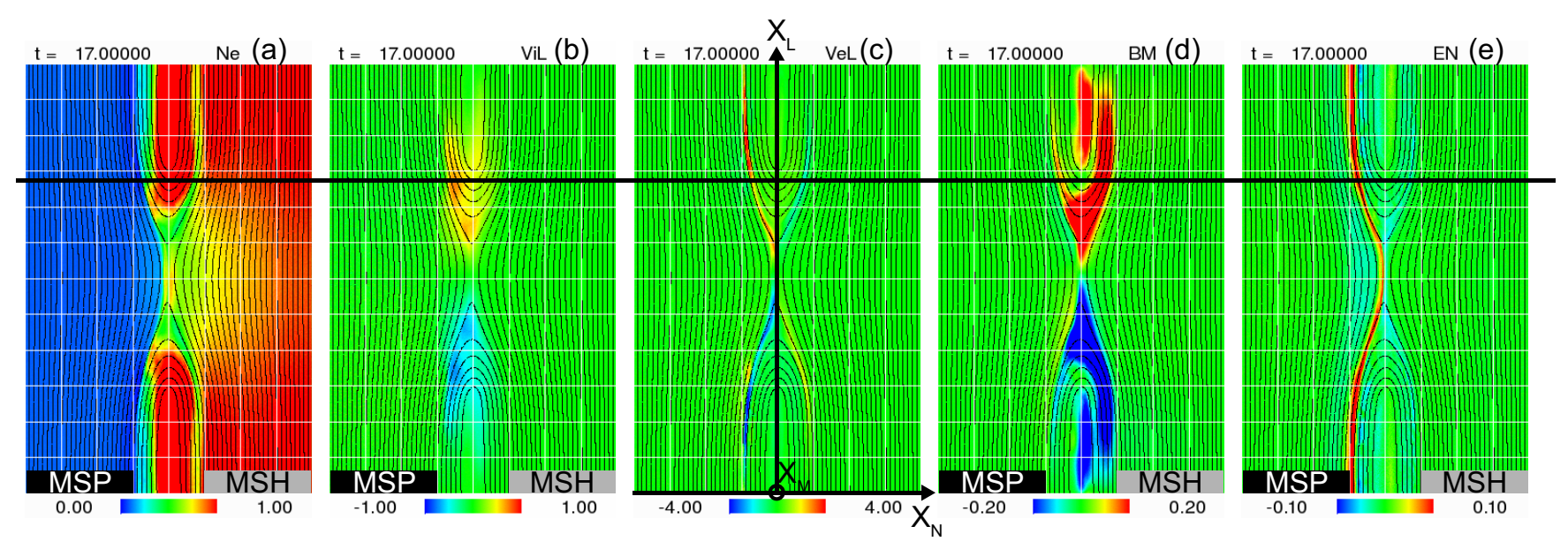

Fig. 4. 2 -D color contours of the simulation results at $t=17$. (a) The electron number density $n_{e}$, (b) the ion outflow bulk velocity $V_{i L}$, (c) the electron outflow bulk velocity $V_{e L}$, (d) the out-of-plane magnetic field $B_{M}$, and (e) the normal component of the electric field $E_{N}$. The bands at the bottom of each panel show the MSP and MSH domain in the simulation box. The horizontal black line shows the virtual spacecraft observation path, along which detailed analysis is made in Fig. 5.

Figure 4 shows the two-dimensional (2-D) color contours of the simulation results at $t=17$. In this figure, (a) the electron number density $n_{e}$, (b) the ion outflow bulk velocity $V_{i L}$, (c) the electron outflow bulk velocity $V_{e L}$, (d) the outof-plane magnetic field $B_{M}$, and (e) the normal component of the electric field $E_{N}$ are shown. A horizontal black line depicts the location $x_{L}=17.625$ (the $\mathrm{X}$-line is at $x_{L}=12$ ), at which the detailed one-dimensional profiles will be inspected later. In this figure, black and light gray bands at the bottom of each panel along $\mathrm{N}$-direction represent the lengths of the MSP, and MSH proper, respectively. The virtual spacecraft is moving from left (MSP) to right (MSH) in the panels. The magnetic field lines are superimposed as black curves on the color contours.

It would be useful to preview with these panels some of the features that will be studied in detail. (1) The density dip on the MSP side will be discussed. In Fig. 4a, while the dip itself is not seen clearly, one can note the density gradient layer to be developed along the separatrix on the MSP side. In contrast, the density dip on the MSH side is clearly seen along the separatrix (This is not clearly seen in the observations). In Fig. 4c, one notes that the separatrix on the MSP side is accompanied by intense electron flow away from the $\mathrm{X}$-line $\left(V_{e L}>0\right)$. (2) In Fig. 4e, a strongly positive $E_{N}$ along the separatrix on the MSP side is found. In contrast $E_{N}$ along the separatrix on the MSH side is rather weak and is negative. (3) In Fig. 4b, the ion flows out from the X-line is shown. At the location of the slice, the ion flow is biased towards the MSP side $x_{N}<0$. This feature has been seen in a hybrid simulation result of Nakamura and Scholer (2000). That is, the size of the present full particle simulation is large enough that such an ion-scale feature can be observed.

While the above three items are used to identify the location $x_{L}=17.625$ at $t=17$ as the best matching virtual space- craft trajectory, the other feature that will be discussed is the out-of-plane magnetic field $B_{M}$ shown in Fig. 4d. If the density is symmetric, $B_{M}$ would show so-called Hall quadrupolar magnetic field. In Fig. $4 \mathrm{~d} B_{M}$ is positive at $x_{L}>0$ and negative for $x_{L}<0$. The electron flow sustaining the corresponding in-plane electric current component flows from the MSH side to the MSP side along the contour of the $B_{M}$ component. This bipolar feature has been seen in a hybrid simulation result of Nakamura and Scholer (2000). An observational study by Øieroset et al. (2004) notes that the same is seen in the distant tail reconnection where north-south density asymmetry is expected.

Figure 5 shows one-dimensional cuts of the simulation results along $x_{L}=17.625,5 \sim 6$ ion inertial length away from the $\mathrm{X}$-line. In Fig. 5, (a) the electron number density, (b) three components of the magnetic fields $B_{L}$ (blue), $B_{M}$ (green), and $B_{N}$ (red), (c) the ion outflow bulk velocity $V_{i L}$. (d) the electron outflow bulk velocity $V_{e L}$, and (e) the normal component of the electric field $E_{N}$ are shown. In this figure, the blue, red, green, and black vertical lines represent the location of density dip, $E_{N}$ peak, $V_{i L}$ peak, and $B_{L}$ reversal, respectively. Those lines are drawn to compare with what was seen in Fig. 1.

Figure 6 shows electron velocity distribution functions at $t=17$ and at $x_{L}=17.625$. Figure $6 \mathrm{a} \sim \mathrm{c}$, and $6 \mathrm{~d} \sim \mathrm{f}$ is the electron distribution function around the density dip on the MSH and MSP sides, respectively. Those electrons are sampled at (a) $x_{N}=1.53$ (the negative peak of $V_{e L}$ ), (b) $x_{N}=1.25$ (the $n_{e}$ dip), (c) $x_{N}=1.44$ (the positive peak of $V_{e L}$ ), and (d) $x_{N}=-1.5$ (the positive peak of $V_{e L}$ ), (e) $x_{N}=-1.67$ (the $n_{e}$ dip), (f) $x_{N}=-1.75$ (the negative peak of $V_{e L}$ ), respectively. The horizontal and vertical axes are the $v_{e N}$ and $v_{e L}$. 
4.2 The structure of the density dip on the magnetospheric side

In Fig. 5a, the electron density is $n_{e}=0.1$ at the outermost side of MSP $x_{N}=-4$. A density dip with $n_{e}=0.04$ is formed around $x_{N}=-1.5$, which is depicted by a blue vertical line. The density depletion rate is $\sim 0.5$ of the outermost MSP level. A large density gradient is observed around $x_{N}=-1.3$. In Fig. 5d, just around the density dip, a negative-to-positive bipolar $V_{e L}$ is seen. There $V_{e L}$ drops down to -0.7 at $x_{N}=-1.7$ and increases up to $V_{e L}=2.7$ at $x_{N}=-1.5$, which is depicted by a red vertical line. In association with the positive $V_{e L}$ peak, $E_{N}$ increases up to 0.12 at $x_{N}=-1.5$.

At the location of the positive peak of $V_{e L}$, most of the electrons are strongly accelerated parallel to the magnetic field, as shown in Fig. 6d. That is, those electrons are flowing away from the X-line. On the contrary, the electrons both at the density dip (Fig. 6e) and negative peak of $V_{e L}$ (Fig. 6f) are weakly accelerated anti-parallel to the magnetic field (into the X-line). The distance between the positive $V_{e L}$ peak (red vertical line) and the $n_{e}$ dip (blue vertical line) is estimated to $\sim 0.2$, which is $\sim 2 c / \omega_{p e}$. Comparison between Fig. $6 \mathrm{~d}$ and e implies that the electron acceleration can be drastically changed within the electron inertial scale in the separatrix region on the MSP side.

The structure of the density dip on the MSP side shows fairly good agreement between simulation and observation. The density at the dip in the simulation is 0.5 times of the MSP level, while in the observation is 0.2 times of the MSP level. At the density dip, both the simulation and observation show the positive $E_{N}$ enhancement. The peak value of $E_{N}$ is about 1.2 , which is in fair agreement with the dimensionless value of 2.3 (based on $40 \mathrm{mV} / \mathrm{m}$ ) observed in the Cluster data. While there is no data of $V_{e L}$ in the observation, the simulation shows the flow reversal of the electron just around the density dip. The mixture of the two populations of accelerated electrons seen in observations at the density dip (see Fig. 2) is suggested to result from low-time resolution sampling.

\subsection{The structure of the ion jet}

In Fig. 5c, the steep lift-off of $V_{i L}$ at $x_{N}=-1.5$ from 0 to the peak value of $V_{i L}=0.6$ at $x_{N}=-1.2$ (depicted by the green vertical line) is seen. Since the center of the magnetic field reversal is located at $x_{N}=0.04$ (the black line), the ion jet is biased toward the MSP side. This structure of the ion jet biased towards the MSP side is seen also in the observation (see the green and black lines in Fig. 1).

4.4 The structure of the density dip on the magnetosheath side

In Fig. 5a, a density dip structure is observed at $x_{N}=1.5$ with $n_{e}=0.6$. The density dip on this MSH side is found to be not

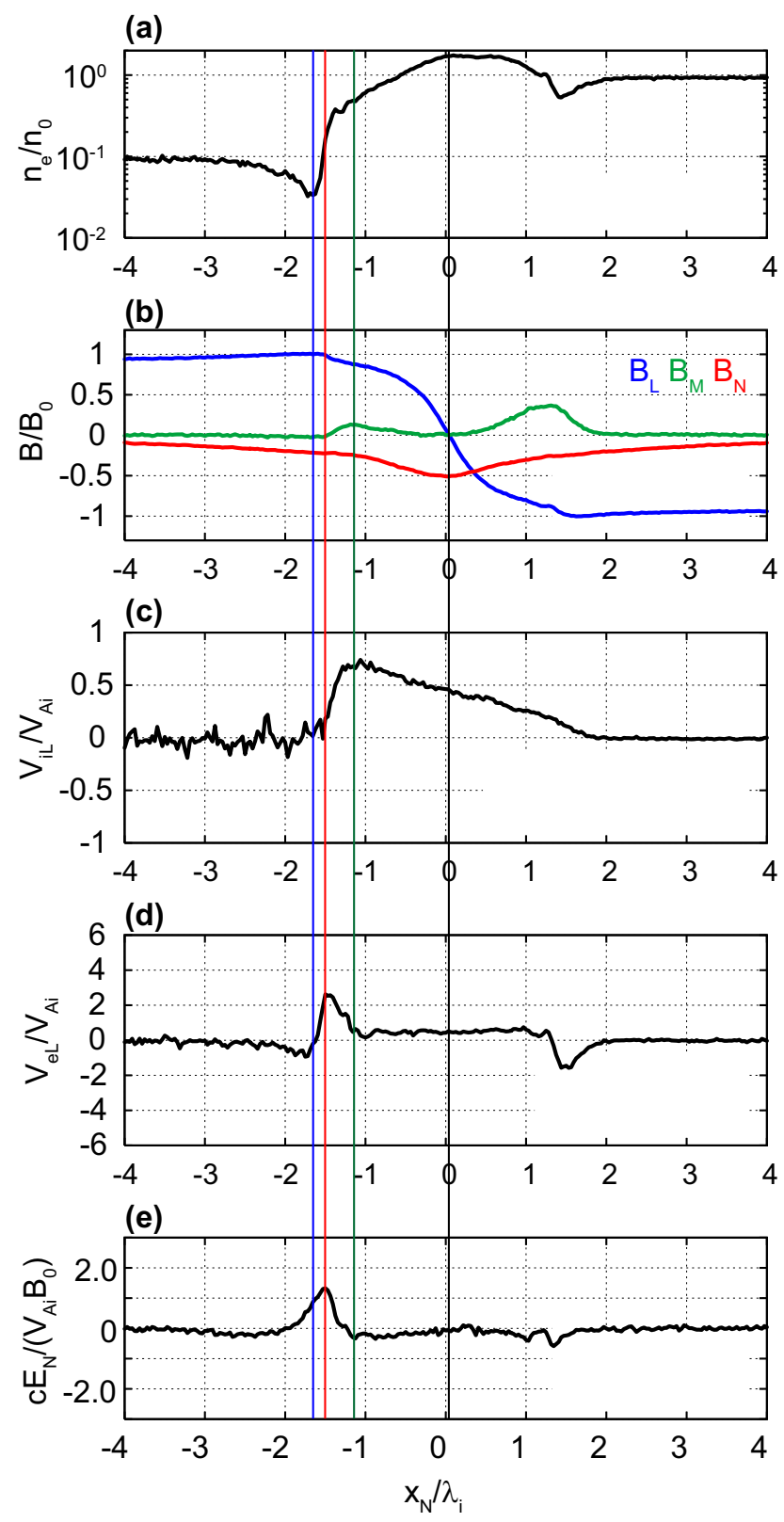

Fig. 5. One-dimensional cuts of the simulation results along $x_{N}$ at $t=17$ at $x_{L}=17.625$. (a) The electron number density, (b) three components of the magnetic fields $B_{L}$ (blue), $B_{M}$ (green), and $B_{N}$ (red), (c) the ion outflow bulk velocity $V_{i L}$. (d) the electron outflow bulk velocity $V_{e L}$, and (e) the normal component of the electric field $E_{N}$. The blue, red, green, and black vertical lines represent the location of the electron density dip, $E_{N}$ peak, $V_{i L}$ peak, and the $B_{L}$ reversal, respectively. These lines correspond to those in Fig. 1.

as deep as that on the MSP side. In Fig. 5d, a well-developed negative electron outflow appears with $V_{e L}=-1.8$ at $x_{N}=1.5$ in association with the density dip. There is a small positive peak of $V_{e L}$ on the MSP side of it. 


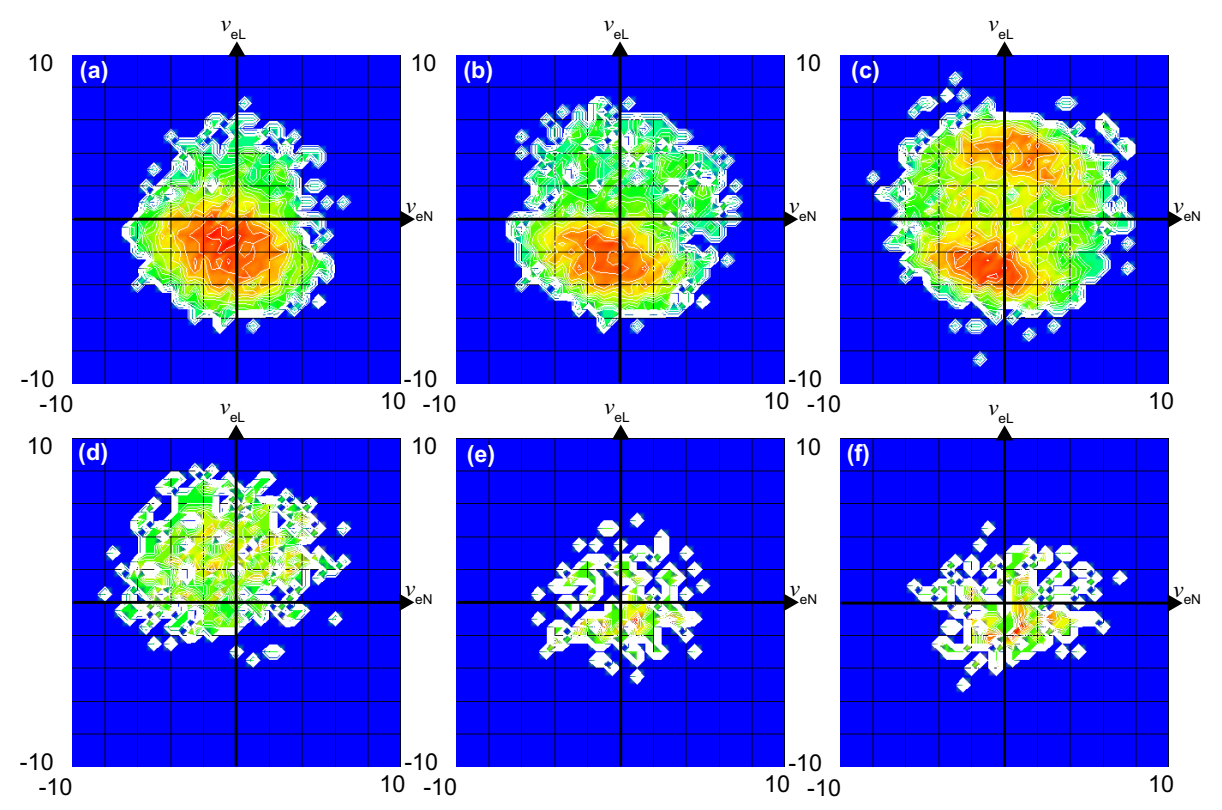

Fig. 6. Electron distribution functions at $t=17$ and at $x_{L}=17.625$. Electrons are sampled at (a) $x_{N}=1.53$ (the negative peak of $V_{e L}$ on the MSH side), (b) $x_{N}=1.25$ (the $n_{e}$ dip on the MSH side), (c) $x_{N}=1.44$ (the positive peak of $V_{e L}$ on the MSH side), (d) $x_{N}=-1.5$ (the positive peak of $V_{e L}$ on the MSP side), (e) $x_{N}=-1.67$ (the $n_{e}$ dip on the MSP side), and (f) $x_{N}=-1.75$ (the negative peak of $V_{e L}$ on the MSP side), respectively. The horizontal and vertical axes are the $v_{e N}$ and $v_{e L}$.

At the location of the negative peak of $V_{e L}$ (Fig. 6a), the electron are accelerated toward the $v_{e N}<0$ and $v_{e L}<0$ direction with their averaged velocity being $v_{e L} \sim-1.6$. At the location of the positive peak of $V_{e L}$ (Fig. 6c), two populations of electron beams coexist in it. One electron beam has a composition of $v_{e N}<0$ and $v_{e L}<0$. This beam seems to be the same electron population as that of Fig. $6 a$ and b. The other electron beam has the velocity of $v_{e N}>0$ and $v_{e L}>0$, and is flowing away from the $\mathrm{X}$-line.

While observation does not detect any clear density dip on the MSH side except for an enhancement of $E_{N}<0$, simulation shows a small density dip in association with $V_{e L}$ flow into the X-line and with the slight $E_{N}$ enhancement on the MSH side. However, the density dip on the MSH side is much smaller than the one on the MSP side, such that it would be quite possible that magnetosheath fluctuations mask its presence.

\subsection{The out-of-plane magnetic field component}

As for the magnetic field structure in Fig. 5b, a small increase of $B_{M}=0.1$ is observed around $x_{N}=-1.2$ on the MSP side. Then a larger increase of $B_{M}=0.4$ is observed at $x_{N}=1.5$ on the MSH side. The asymmetric $B_{M}>0$ signature can quite well reproduce the observation in Fig. 1b. This asymmetric is different from the quadrupolar pattern expected during symmetric reconnection (e.g. Pritchett, 2001; Shay et al., 2001). Nakamura and Scholer (2000) have already shown the $B_{M}$ asymmetry. They have explained that the much stronger $B_{M}$ on the MSH side results from the much higher plasma density on the MSH side. We will show an alternative interpretation later. Comparison between simulation and observation shows that $B_{M}$ peak at the MSH side is 4 times larger than that at the MSP side in the simulation. Meanwhile, in the observation, $B_{M}$ peak at the MSH side is 2.5 times larger than that at the MSP side.

\section{Electron acceleration and the magnetic field structure}

In the previous section, comparison between simulation and observation has been made with respect to the key features. While the comparison along a one-dimensional cut, which showed rather good agreement, is a good validation of the simulation, a deeper analysis in the two-dimensional simulation results would give us a clearer view of what physical processes lead to the observed features. In this section, we will first inspect the spatial structure of the magnetic field. Then we will realize that the enhanced $E_{N}$ at the MSP SR, which was independently picked-up as a key feature in the observations, is the origin of the bipolar Hall $B_{M}$. Furthermore we will see that the electron beam away from the X-line detected at the MSP SR is related to the bipolar $B_{M}$. That is, the key features identified in the observations are found to be strongly related with each other. These findings clearly show how the simulation results can complement spacecraft observations by providing extra information not available via observations. 


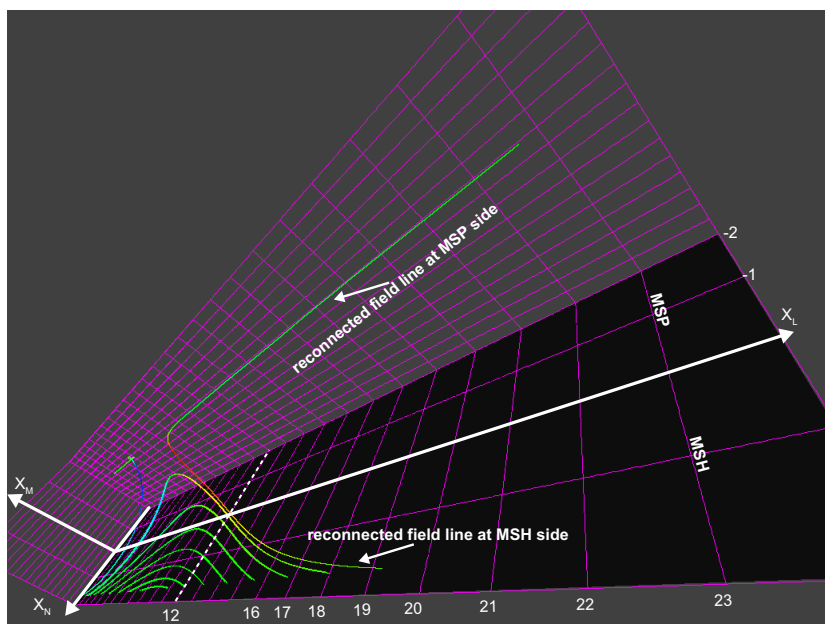

Fig. 7. Three-dimensional structure of the magnetic field lines. Color on the field lines represents $B_{M}$. Color of red, green, and blue in the field lines are $B_{M}>0, \sim 0$, and $<0$, respectively. Focusing on a newly reconnected magnetic field, this reconnected field line is strongly lifted up in the positive $x_{M}$-direction around the $\mathrm{X}$ line on the MSH side where $B_{M}>0$ is strongly enhanced. Then, it lies on the $x_{L}-x_{N}$ plane along the MSP SR $\left(B_{M} \sim 0\right)$. This deformation of the field line takes place as the portion of the reconnected field lines along the MSP SR quickly drifts in the $+\mathrm{M}$ direction upon crossing the SR.

\subsection{The origin of the bipolar Hall magnetic field}

The mono-signed $B_{M}$ pattern in Fig. $4 \mathrm{~d}$ implies that the magnetic field lines within the jet are slanted towards the $+\mathrm{x}_{M}$ direction in the M-N plane. Noting that $B_{N}$ is negative on this side of the X-line, this bending of the field lines can be made if the part of the reconnected field line situated at the MSP side edge is pulled to the $+\mathrm{M}$ direction. To show that this is the case, the three-dimensional structure of the magnetic field lines is shown in Fig. 7.

Color on the field lines represents $B_{M}$ at each location. Red, green, and blue are $B_{M}>0, \sim 0$, and $<0$, respectively. Field lines with substantial $B_{M}$ appear to be bent toward the $x_{M}$-direction. On the contrary, field lines with $B_{M} \sim 0$ stay on the $x_{L}-x_{N}$ plane. Let us focus on a newly reconnected magnetic field at $x_{L}>12$. This reconnected field line is strongly lifted up in the positive $x_{M}$-direction around the $\mathrm{X}$-line on the MSH side where $B_{M}>0$ is strongly enhanced. Then, it lies on the $x_{L}-x_{N}$ plane along the MSP SR. This deformation of the field line takes place as the portion of the reconnected field lines along the MSP SR quickly drifts in the $+\mathrm{M}$ direction upon crossing the SR.

In Fig. 8, comparison between $B_{M}$ and the M-component of the $\boldsymbol{E} \times \boldsymbol{B}$-drift $V_{\boldsymbol{E} \times \boldsymbol{B}, M}$ is shown. Along the MSP SR, the enhanced $E_{N}$, which was discussed before (Fig. 4e), produces an enhancement in $V_{\boldsymbol{E} \times \boldsymbol{B}, M}>0$. This means that the reconnected field are shifted in the $+\mathrm{M}$ direction upon crossing the SR and this happens only on the MSP side. A consid-
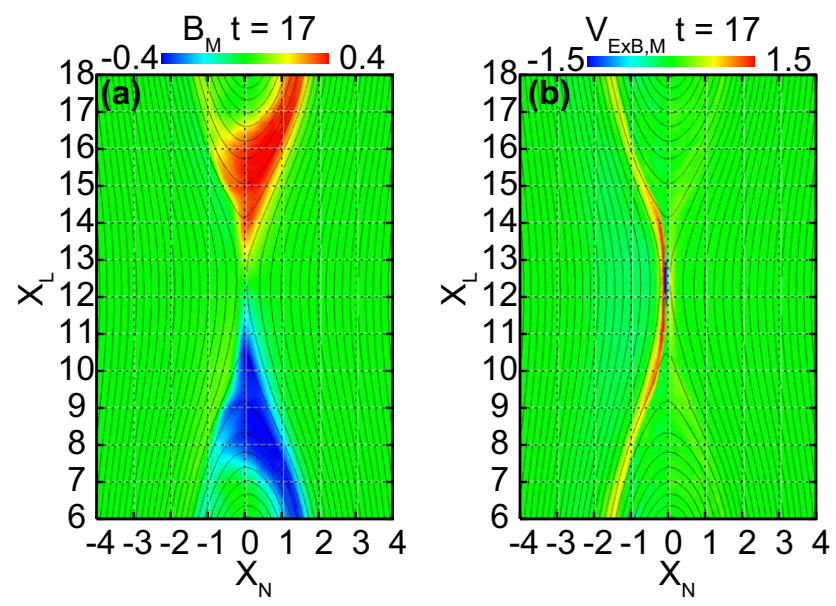

Fig. 8. Comparison between (a) $B_{M}$ and (b) $V_{\boldsymbol{E} \times \boldsymbol{B}, M}$. Along the MSP SR, the enhanced $E_{N}$ makes enhanced $V_{\boldsymbol{E} \times \boldsymbol{B}, M}>0$ to be present. This means that the reconnected field are shifted in the $+\mathrm{M}$ direction upon crossing the SR and this happens only on the MSP side.

eration of how the field lines behave in the $\mathrm{M}-\mathrm{N}$ plane makes us understand why only positive $B_{M}$ is seen within the jet on this side of the $\mathrm{X}$-line, and negative $B_{M}$ on the other side.

\subsection{Electron beam at the magnetospheric side separatrix}

It is interesting to see from our simulation results how the electron beam away from the X-line at the MSP SR is produced during magnetic reconnection. In Fig. 9, we inspected the electron velocity distribution functions along a reconnected magnetic field line. Figure $9 \mathrm{a}$ is the 2-D contour of $V_{e M}$ at $t=17$. The enhanced electron flow in the $+\mathrm{M}$ direction is seen at the reconnection region and extends along the MSP SR. Figure $9 \mathrm{~b} \sim \mathrm{e}$ is the electron distribution function at the several locations marked in Fig. 9a by the blue asterisks. Red lines in Fig. 9c e show the directions of the local magnetic field at each location. Each red line in Fig. 9c $\sim$ e is given by $v_{M}=\left(B_{M} / B_{L}\right) \times\left(v_{L}-V_{\boldsymbol{E} \times \boldsymbol{B}, L}\right)+V_{\boldsymbol{E} \times \boldsymbol{B}, M}$, where $B_{M}, B_{L}, V_{\boldsymbol{E} \times \boldsymbol{B}, L}$, and $V_{\boldsymbol{E} \times \boldsymbol{B}, M}$ are the values at each sampling location. The slope of the red line is the direction of magnetic field in L-M plane at each location. In Fig. 9b, we do not draw a red line since $|\mathbf{B}|$ at that location is too small. The point $\left(v_{L}, v_{M}\right)=\left(V_{\boldsymbol{E} \times \boldsymbol{B}, L}, V_{\boldsymbol{E} \times \boldsymbol{B}, M}\right)$ is the origin to the observer who is moving with the $\boldsymbol{E} \times \boldsymbol{B}$-drifting field line. Figure $9 b$ and $c$ shows that significant electron acceleration in the $+\mathrm{M}$ direction in the close proximity of the $\mathrm{X}$-line has substantial component along the field line because $B_{M}$ is non-zero (and positive) even at the center of the jet. The field aligned electrons simply stream along the field line. With increasing distance from the $\mathrm{X}$-line, the magnetic field rotates towards the L-axis (Fig. 9d and e). Together with this, the electron distribution function shape also rotates but this only reflects the fact that field-aligned component stays field aligned as they stream away from the X-line. 

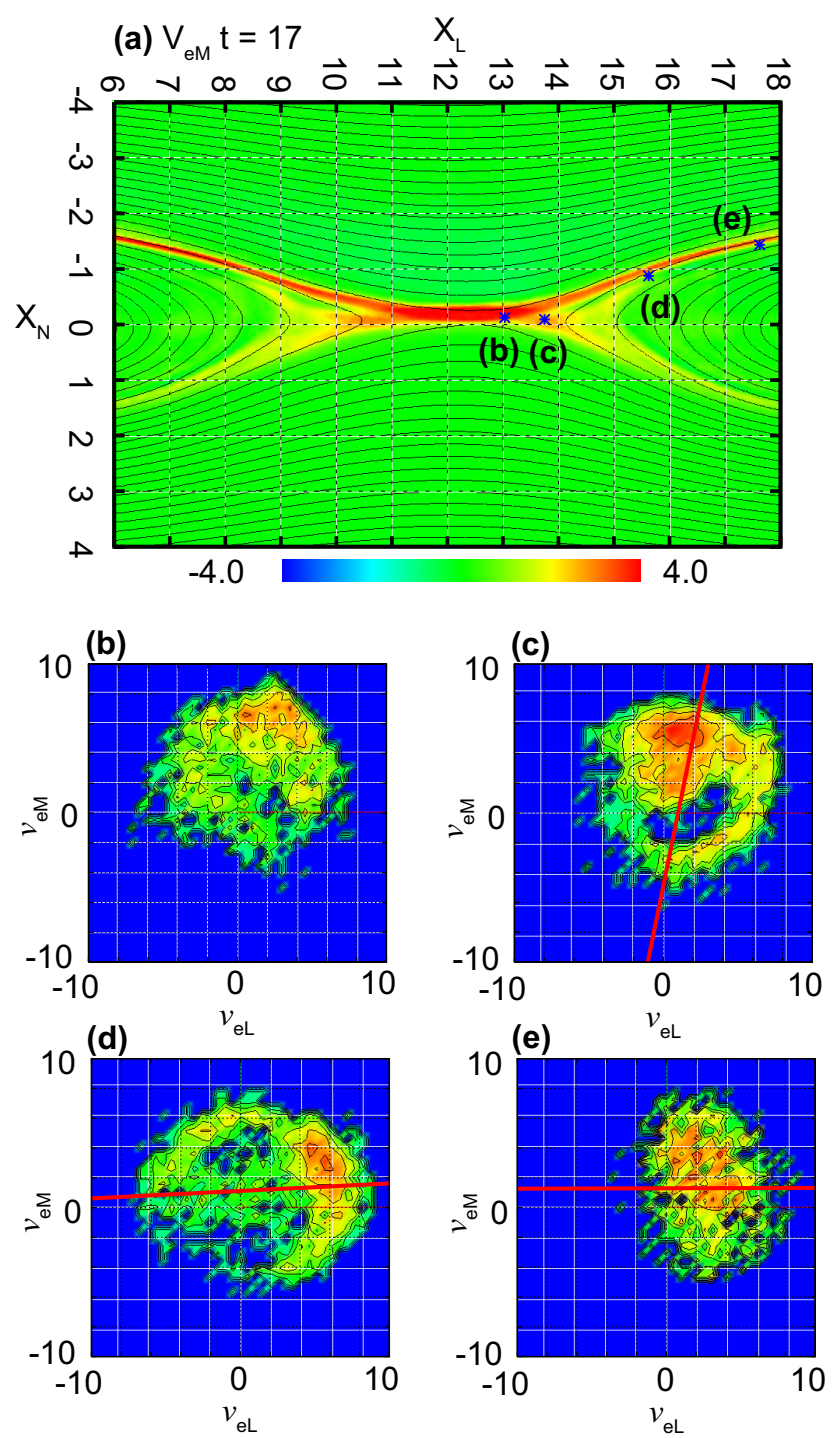

Fig. 9. The origin of the electron beam along the separatrix on the magnetosphere side. (a) 2-D contour of $V_{e M}$. (b) $\sim$ (e) Electron distribution functions on the $v_{N}=0$ plane. The sampling locations are $\left(x_{N}, x_{L}\right)=(-0.13,13),(-0.1,13.75),(-0.9,15.6)$, and $(-1.5,17.6)$. The red lines in Fig. $9 \mathrm{c} \sim \mathrm{e}$ is the direction of the local magnetic field at each location. Each red line in Fig. 9c $\sim \mathrm{e}$ is given by $v_{M}=\left(B_{M} / B_{L}\right) \times\left(v_{L}-V_{\boldsymbol{E} \times \boldsymbol{B}, L}\right)+V_{\boldsymbol{E} \times \boldsymbol{B}, M}$, where $B_{M}$, $B_{L}, \mathrm{~V}_{\boldsymbol{E} \times \boldsymbol{B}, L}$, and $V_{\boldsymbol{E} \times \boldsymbol{B}, M}$ are the values at each sampling location. The slope of the red line is the direction of magnetic field in L$M$ plane at each location. The point $\left(v_{L}, v_{M}\right)=\left(V_{\boldsymbol{E} \times \boldsymbol{B}, L}, V_{\boldsymbol{E} \times \boldsymbol{B}, M}\right)$ is the origin for an observer that is co-moving with the field line.

The origin of the MSP SR electron beam would be summarized as follows: In the center of the current sheet, strong electron acceleration toward the $+\mathrm{M}$-direction is attained as in any reconnection geometry. The density asymmetry, however, makes $B_{M}$ not zero at the center of the current sheet and accelerated electrons are field aligned. They simply remain field aligned until they are detected at the SR. It is clear that the bipolar Hall field (and thus, as describe above, the enhanced $E_{N}$ at the MSP SR) is the crucial factor for the formation of the beam at the MSP SR.

\section{Discussion}

In the present paper, we have carried out a comparative study between the magnetopause crossing observations by Cluster and a two-dimensional particle-in-cell simulation. The MP crossing event had a substantial density jump across the boundary but was without guide field and thus is a good chance to study the effects of the density asymmetry across a current sheet on magnetic reconnection.

Regarding observation-simulation comparison, fairly good agreement is achieved. (1) The structure of the density dip on the magnetospheric side is fairly in good agreement between simulation and observation. Both the simulation and observation show the positive $E_{N}$ enhancement. While the observation shows a mixture of two populations of the accelerated electrons at the density dip, the simulation suggests that the mixture may result from low-time resolution of the measurements. (2) The peak location of the ion jet velocity is biased toward the MSP side in both the simulation and the observation. (3) The out-of-plane magnetic field component shows good correspondence between our simulation and the observation. The out-of-plane magnetic field on the MSH side is larger than that on the MSP side.

Meanwhile, on the MSH side, the structure of the density dip is different. In the simulation, the MSH side SR forms an evidently visible density dip with weak but certain $V_{e L}$ and $E_{N}$ signatures like the MSP case. In contrast, observation do not show a clear density dip at the MSH side. The explanation for the discrepancy could be that the structure may be too weak to stand out against the fluctuations in the magnetosheath.

We have stepped beyond a simple observation-simulation comparison and studied the simulation results to find that the key features of the asymmetric reconnection that we identified in the observations are indeed related to each other. The out-of-plane $\boldsymbol{E} \times \boldsymbol{B}$-drift due to the enhanced $E_{N}$ (the key feature \#1) at MSP SR bends the field lines such the bipolar $B_{M}$ (key feature \#2) appears in the reconnection jet. Then this $B_{M}$ makes the electrons accelerated at the $\mathrm{X}$-line to have substantial field-aligned component. These electrons simply stream along the MSP SR to be detected as the electron beam there (key feature \#3).

The best matching with the observations in the simulation box was obtained at 5 6 ion inertial length from the $\mathrm{X}$-line. This is much smaller than the $\sim 50$ inertial lengths found by Retinò et al. (2006) by comparing with another numerical simulation by Bavassano Cattaneo et al. (2006). The thickness of the reconnection layer at this location is about 3 ion inertial lengths. Meanwhile, Cluster observed the $B_{L}$ rotation from 10:57:54 to 10:58:20 UT. Assuming that $1 \mathrm{~s}$ 
(a)

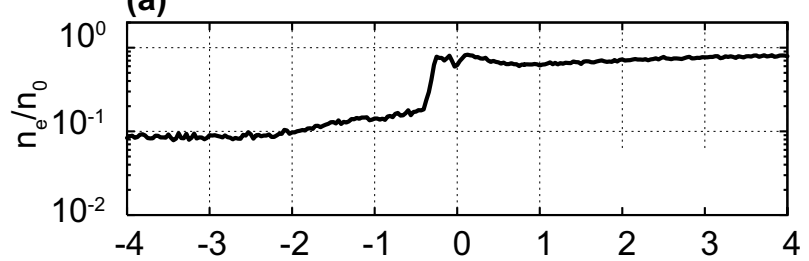

(b)

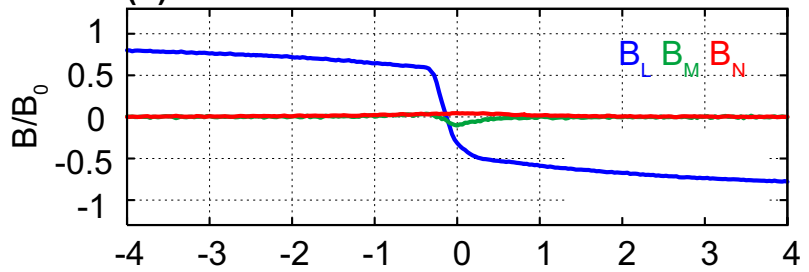

1 (c)

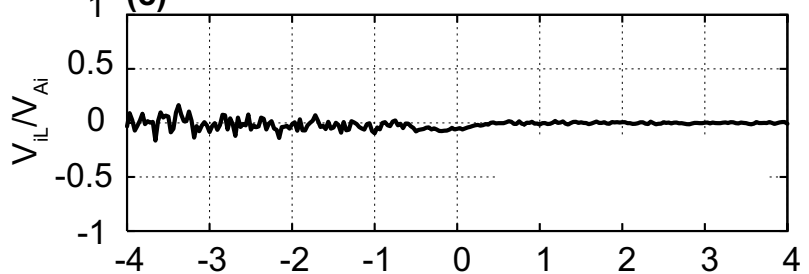

(d)
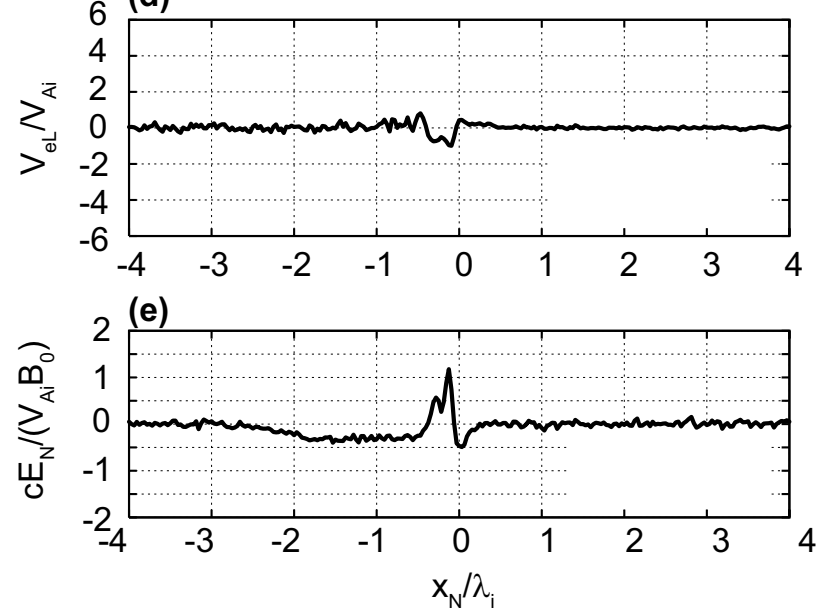

Fig. 10. The vertical structure of the $X$-line $\left(x_{L}=12\right)$ under the density asymmetry. The format is the same as Fig. 5.

corresponds to $20 \mathrm{~km} \sim 0.5$ ion inertial length (Retinò et al., 2006) the current sheet thickness is $\sim 13$ ion inertial length. The difference by a factor of $\sim 4$ may be taken to be within the range of the error bar associated with the ambiguity in the observations. It is also noted that temporal features such as the passage of magnetic islands, which are not included in the simulation but are observed by Retinò et al. (2006) can account for this discrepancy. On the other hand, the observation location might be further from the X-line by a factor of $\sim 4$, which is $\sim 20$ ion inertial lengths away from the $X$-line. While the simulation in such a larger box for an extended time is not available now, fairly good agreement obtained

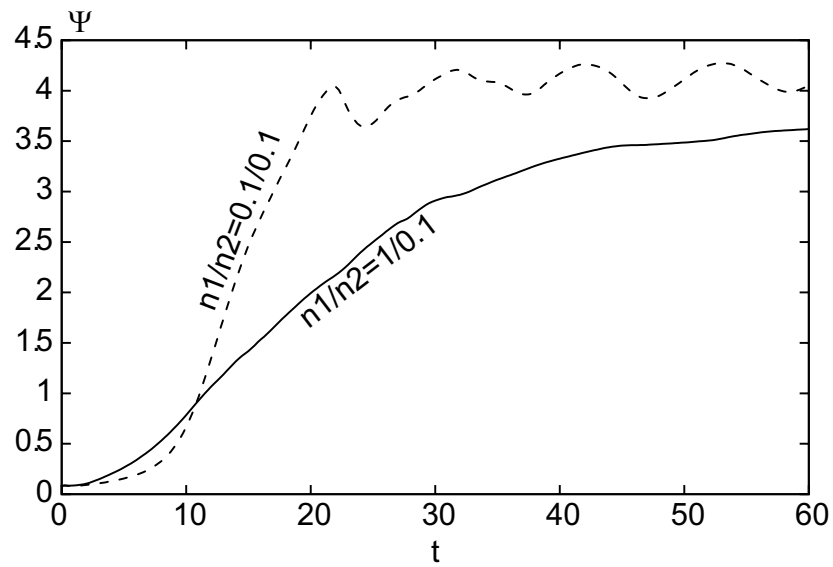

Fig. 11. Comparison between the present case (solid black), and the symmetric $n_{1} / n_{2}=0.1 / 0.1$ case (dashed black). Reconnected magnetic flux $\Psi$ as a function of time is shown. $\Psi$ of the $n_{1} / n_{2}=0.1 / 0.1$ case shows faster reconnection rate and larger saturation level.

here suggest that the spatial structure studied here will expand self-similarly to show the essentially the same features at the new location more distant from the X-line.

As indicated in Table 1, the observed ion and electron temperatures in the MSP and in the MSH are similar. This is typical of high-latitude lobe reconnection (i.e. reconnection between MSH and MSP lobe field lines tailward of the magnetospheric cusp) and differs from low-latitude reconnection occurring between cold MSH and hot plasma sheet plasma. In both cases, however, there is usually a large density asymmetry between MSH and MSP. In this paper, we have focused on the effects of the density gradient during MP reconnection rather than on the temperature gradient. However in the simulation, $T_{i}$ in the MSP is 19 times larger than in the MSH suggesting that temperature gradients are also included in the present results. The role of temperature asymmetry requires further analysis and will be investigated in another study.

Another relevant issue that is not included here is the velocity shear between MSP and MSH. While our simulation has no velocity shear across the MP, the observations show that the outermost magnetosheath flow velocity is $V_{s h} \sim 150 \mathrm{~km} / \mathrm{s}$ (at 11:00:00 UT in Fig. 1 of Retinò et al., 2006). With the MSH ion Alfvén speed at $V_{A, s h, i} \sim 240 \mathrm{~km} / \mathrm{s}$, the Alfvén Mach number of the sheath flow is $\sim 0.63$. Inspection of our very recent MP reconnection simulation run which includes the sheath flow of $V_{s h}=0.5 V_{A, s h, i}$ indicates that the key features studied in this paper are only slightly modified by this moderate Mach number flow. Details will be given in a future publication.

In the simulation runs, we are able to sample the reconnection layers at locations which a spacecraft seldom could cross. In Fig. 10, the one-dimensional structure at the X-line is shown. The format is the same as Fig. 5. In this figure, a steep density gradient is detected at $x_{N}=-0.3$. While it is 
stable in the present 2-D run, in the three-dimensional (3-D) situation it may be unstable to the lower-hybrid drift instability (LHDI) (Davidson et al., 1977). This may bring another feature to appear in magnetic reconnection under a density asymmetry and it will be investigated in the future.

As was described in the Introduction, our study concentrates on the electron dynamics embedded in a reconnecting layer with density asymmetry. An important issue is how the density asymmetry modifies the reconnection rate. In Fig. 11, a comparison between different density ratios $n_{1} / n_{2}=1 / 0.1$ (solid black), $n_{1} / n_{2}=0.1 / 0.1$ (dashed black) cases is made. In this figure, the temporal development of reconnected magnetic flux is shown. Evidently, the case with $n_{1} / n_{2}=0.1 / 0.1$ has both faster growth rate and the larger saturation level. It is interesting to see how the reconnection rate is controlled or scaled by $n_{1} / n_{2}$, and this will be discussed in a future work.

Acknowledgements. Numerical simulations are performed on NEC SX6 at JAXA. We thank the referees for their assistance in evaluating this paper. K. G. Tanaka was supported by the Grant-in-Aid for Creative Scientific Research "The Basic Study of Space Weather Prediction" (17GS0208, Head Investigator: K. Shibata) from the Ministry of Education, Science, Sports, Technology, and Culture of Japan. K. G. Tanaka was also supported by Grant-in-Aid for JSPS Fellows (20.9859), from Japan Society for the Promotion of Science. The work done by A. Retinò at the Swedish Institute of Space Physics was supported by the Swedish National Space Board.

Topical Editor I. A. Daglis thanks two anonymous referees for their help in evaluating this paper.

\section{References}

André, M., Vaivads, A., Buchert, S. C., Fazakerley, A. C., and Lahiff, A.: Thin electron-scale layers at the magnetopause, Geophys. Res. Lett., 31, L03803, doi:10.1029/2003GL018137, 2004.

Bavassano Cattaneo, M. B., Marcucci, M. F., Retinò, A., Pallocchia, G., Rème, H., Dandouras, I., Kistler, L. M., Klecher, B., Carlson, C. W., Korth, A., McCarthy, M., Lundin, R., and Balogh, A.: Kinetic signatures during a quasi-continuous lobe reconnection event: Cluster Ion Spectrometer (CIS) observations, J. Geophys. Res., 111, A09212, doi:10.1029/2006JA011623, 2006.

Borovsky, J. E. and Hesse, M.: The reconnection of magnetic fields between plasmas with different densities: Scaling relations, Phys. Plasmas, 14, 102309, doi:10.1063/1.1772619, 2007.

Cassak, P. A. and Shay, M. A.: Scaling of asymmetric magnetic reconnection: General theory and collisional simulations, Phys. Plasmas, 14, 102114, doi:10.1063/1.2795630, 2007.

Daughton, W., Lapenta, G., and Ricci, P.: Nonlinear Evolution of the Lower-Hybrid Instability in a Current Sheet, Phys. Rev. Lett., 93, 105004, doi:10.1103/PhysRevLett.93.105004, 2004.

Davidson, R. C., Gladd, N. T., Wu, C. S., and Huba, J. D.: Effects of finite plasma beta on the lower-hybrid-drift instability, Phys. Fluids, 20, 301-310, 1977.

Frey, H. U., Phan, T. D., Fuselier, S. A., and Mende, S. B.: Continuous magnetic reconnection at Earth's magnetopause, Nature, 426, 533-537, 2003.
Fuselier, S. A., Trattner, K. J., Petrinec, S. M., Owen, C. J., and Rème, H.: Computing the reconnection rate at the Earth's magnetopause using two spacecraft observations, J. Geophys. Res., 110, A06212, doi:10.1029/2004JA010805, 2006.

Gary, S. P. and Sgro, A. G.: The lower hybrid drift instability at the magnetopause, Geophys. Res. Lett., 17, 909-912, 1990.

Haijima, K., Tanaka, K. G., Fujimoto, M., and Shinohara, I.: Electron temperature anisotropy effects on tearing mode in ion-scale current sheets, Adv. Space Res., 41, 1643, doi:10.1016/j.asr.2007.05.049, 2008.

Harris, E. G.: On a plasma sheath separating regions of oppositely directed magnetic field, Nuovo Cimento, 23, 115-121, 1962.

Hoshino, M.: The electrostatic effect for the collisionless tearing mode, J. Geophys. Res., 93, 7368-7380, 1987.

Mozer, F. S., Bale, S. D., and Phan, T. D.: Evidence of Diffusion Regions at a Subsolar Magnetopause Crossing, Phys. Rev. Lett., 89, 015002, doi:10.1103/PhysRevLett.89.015002, 2002.

Nakamura, M. and Scholer, M.: Structure of the magnetopause reconnection layer and of flux transfer events: Ion kinetic effects, J. Geophys. Res., 105, 23 179-23 191, 2000.

Øieroset, M., Phan, T. D., and Fujimoto, M.: Wind observations of asymmetric magnetic reconnection in the distant magnetotail, Geophys. Res. Lett., 31, L12801, doi:10.1029/2004GL019958, 2004.

Panov, E. V., Büchner, J., Fränz, M., Korth, A., Khotyaintsev, Y., Nikutowski, B., Savin, S., Fornaçon, K.-H., Dandouras, I., and Rème, H.: CLUSTER spacecraft observation of a thin current sheet at the Earth's magnetopause, Adv. Space Res., 37, 13631372, doi:10.1016/j.asr.2005.08.024, 2005.

Phan, T. D., Frey, H. U., Frey, S., Peticolas, L., Fuselier, S., Carlson, C., Rème, H., Bosqued, J.-M., Balogh, A., Dunlop, M., Kistler, L., Mouikis, C., Dandouras, I., Sauvaud, J. -A., Mende, S., McFadden, J., Parks, G., Moebius, E., Klecker, B., Paschmann, G., Fujimoto, M., Petrinec, S., Marcucci, M. F., Korth, A., and Lundin, R.: Simultaneous Cluster and IMAGE observations of cusp reconnection and auroral proton spot for northward IMF, Geophys. Res. Lett., 30, 1509, doi:10.1029/2003GL016885, 2003.

Paschmann, G., Sonnerup, B. U. Ö., Papamastorakis, I., Sckopke, N., Haerendel, G., Bame, S. J., Asbridge, J. R., Gosling, J. T., Russell, C. T., and Elphic, R. C.: Plasma acceleration at the Earth's magnetopause: Evidence for reconnection, Nature, 282, 243-246, doi:10.1038/282243a0, 1979.

Phan, T. D., Kistler, L. M., Klecher, B., Haerendel, G., Paschmann, G., Sonnerup, B. U. Ö., Baumjohann, W., Bavassano-Cattaneo, M. B., Carlson, C. W., DiLellis, A. M., Fornacon, K. -H., Frank, L. A., Fujimoto, M., Gworgescu, E., Kokubun, S., Moebius, E., Mukai, T., Øieroset, M., Paterson, W. R., and Rème, H.: Extended magnetic reconnection at the Earth's magnetopause from detection of bi-directional jets, Nature, 404, 848-850, doi:10.1038/35009050, 2000.

Pritchett, P. L.: Geospace Environment Modeling magnetic reconnection challenge: Simulations with a full particle electromagnetic code, J. Geophys. Res., 106(A3), 3783-3798, 2001.

Retinò, A., Bavassano Cattaneo, M. B., Marcucci, M. F., Vaivads, A., André, M., Khotyaintsev, Y., Phan, T., Pallocchia, G., Rème, H., Möbius, E., Klecker, B., Carlson, C. W., McCarthy, M., Korth, A., Lundin, R., and Balogh, A.: Cluster multispacecraft observations at the high-latitude duskside magnetopause: impli- 
cations for continuous and component magnetic reconnection, Ann. Geophys., 23, 461-473, 2005, http://www.ann-geophys.net/23/461/2005/.

Retinò, A., Vaivads, A., André, M., Sahraoui, F., Khotyaintsev, Y., Pickett, J. S., Bavassano Cattaneo, M. B., Marcucci, M. F., Morooka, M., Owen, C. J., Buchert, S. C., and Comilleau-Wehrlin, N.: Structure of the separatrix region close to a magnetic reconnection X-line: Cluster observations, Geophys. Res. Lett., 33, L06101, doi:10.1029/2005GL024650, 2006.

Pinnock, M., Chisham, G., Coleman, I. J., Freeman, M. P., Harston, M., and Villain, J.-P.: The location and rate of dayside reconnection during an interval of southward interplanetary magnetic field, Ann. Geophys., 21, 1467-1482, 2003, http://www.ann-geophys.net/21/1467/2003/.

Ricci, P., Brackbill, J. U., Daughton, W., and Lapenta, G.: Influence of the lower-hybrid drift instability on the onset of magnetic reconnection, Phys. Plasmas, 11, 4489-4500, doi:10.1063/1.1778744, 2004.

Scholer, M., Sidorenko, I., Jaroschek, C. H., Treumann, R. A., and Zeiler, A.: Onset of collisionless magnetic reconnection in thin current sheets: Three-dimensional particle simulations, Phys. Plasmas, 10, 3521-3527, doi:10.1063/1.1597494, 2003.

Shay, M. A., Drake, J. F., Rogers, B. N., and Denton, R. E.: Alfvénic collisionless magnetic reconnection and the Hall term, J. Geophys. Res., 106(A3), 3759-3772, 2001.

Shinohara, I. and Fujimoto, M.: Formation of thin electron current layer associated with lower hybrid drift instability and its relation to quick reconnection triggering, in: Frontiers of Magnetospheric Plasma Physics, COSPAR Colloq. Ser., vol. 16, edited by: Hoshino, M., Omura, Y., and Lanzerotti, L., pp. 123-129, Elsevier, New York, 2005.
Silin, I. and Büchner, J.: Three-dimensional Vlasov-code simulations of magnetopause-like current sheets, Adv. Space Res., 37, 1354-1362, 2006.

Stenberg, G., Oscarsson, T., André, M., Vaivads, A., Morooka, M., Cornilleau-Wehrlin, N., Fazakerley, A., Lavraud, B., and Décréau, P. M. E.: Electron-scale sheets of whistlers close to the magnetopause, Ann. Geophys., 23, 3715-3725, 2005, http://www.ann-geophys.net/23/3715/2005/.

Swisdak, M., Rogers, B. N., Drake, J. F., and Shay, M. A.: Diamagnetic suppression of component magnetic reconnection at the magnetopause, J. Geophys. Res., 108(A5), 1218, doi:10.1029/2002JA009726, 2003.

Tanaka, K. G., Shinohara, I., and Fujimoto, M.: Effects of guide field on quick magnetic reconnection triggering, Geophys. Res. Lett., 31, L22806, doi:10.1029/2004GL021191, 2004.

Tanaka, K. G., Shinohara, I., and Fujimoto, M.: Quick magnetic reconnection triggering in an ion-scale current sheet: Transition from Type-I to Type-II, Geophys. Res. Lett., 32, L17106, doi:10.1029/2005GLL023718, 2005.

Tanaka, K. G., Shinohara, I., and Fujimoto, M.: Parameter dependence of quick magnetic reconnection triggering: A survey study using two-dimensional simulations, J. Geophys. Res., 111, A11S18, doi:10.1029/2006JA011968, 2006.

Trattner, K. J., Mulcock, J. S., Petrinec, S. M., and Fuselier, S. A.: Probing the boundary between antiparallel and component reconnection during southward interplanetary magnetic field conditions, J. Geophys. Res., 112, A08210, doi:10.1029/2007JA012270, 2007. 